M. S. Rahman*, M. A. Mahmud, H. R. Pota, M. J. Hossain and T. F. Orchi

\title{
Distributed Multi-Agent-Based Protection Scheme for Transient Stability Enhancement in Power Systems
}

\begin{abstract}
This paper presents a new distributed agentbased scheme to enhance the transient stability of power systems by maintaining phase angle cohesiveness of interconnected generators through proper relay coordination with critical clearing time (CCT) information. In this distributed multi-agent infrastructure, intelligent agents represent various physical device models to provide dynamic information and energy flow among different physical processes of power systems. The agents can communicate with each other in a distributed manner with a final aim to control circuit breakers (CBs) with CCT information as this is the key issue for maintaining and enhancing the transient stability of power systems. The performance of the proposed scheme is evaluated on a standard IEEE 39-bus New England benchmark system under different large disturbances such as three-phase short-circuit faults and changes in loads within the systems. From the simulation results, it is found that the proposed scheme significantly enhances the transient stability of power systems as compared to a conventional scheme of static CB operation.
\end{abstract}

Keywords: power system, multi-agent system, transient stability, critical clearing time, protection relays

DOI 10.1515/ijeeps-2014-0143

\footnotetext{
*Corresponding author: M. S. Rahman, School of Engineering and Information Technology, University of New South Wales, PO Box 7916, Canberra, BC 2610, Australia, E-mail: md.rahman3@student. adfa.edu.au

M. A. Mahmud, School of Software and Electrical Engineering, Swinburne University of Technology, Hawthorn, VIC 3122, Australia, E-mail: mmahmud@swin.edu.au

H. R. Pota, School of Engineering and Information Technology, University of New South Wales, PO Box 7916, Canberra, BC 2610, Australia, E-mail: h.pota@adfa.edu.au

M. J. Hossain, Griffith School of Engineering, Griffith University, Gold Coast, QLD 4222, Australia, E-mail: j.hossain@griffith.edu.au T. F. Orchi, School of Software and Electrical Engineering, Swinburne University of Technology, Hawthorn, VIC 3122, Australia, E-mail: orchi.fahima@gmail.com
}

\section{Introduction}

Power system stability, especially transient stability, has increasingly become an area of concern from the early days of operation as power systems over large geographical areas are interconnected using a complex array of devices. Moreover, as they exhibit continuously changing behaviors due to changes in loads or external disturbances, it is essential to balance the overall system operation against these changes so that power system stability is maintained. In such situations, it is essential to settle a power system to either its original or new operating states within the critical clearing time (CCT) which is the maximum time by which a disturbance must be cleared in order to preserve the stability of power systems [1].

The CCT indicates robustness of power systems in terms of transient stability enhancement and the process of transient stability analysis with the CCT information is indispensable as this type of stability is concerned with severe disturbances [2, 3]. Thus, the calculation of accurate CCT is a vital factor for faster transient stability assessment. Some hybrid methods, which are the combination of direct or transient energy function and timedomain simulations, have been proposed in [4-6] to calculate the CCT for online transient stability improvement. In fact, these methods for CCT calculation are mainly based on the consideration of fixed nominal loads connected to the generators though the CCT is adversely affected through sudden changes in loads. In $[7,8]$, the CCT has been calculated using a centralized approach with time-domain simulation to enhance the transient stability of power systems. But in practice, power systems are interconnected systems and composed of different utilities amongst which there is an agreement on performance standards where each utility is free to choose the way to prevent the system from possible disastrous consequences.

With the advanced technologies, the centralized structure has changed to distributed one for the distributed automation system to enhance the operating efficiency [9]. The complexities in power systems and 
difficulties in centralized approaches have motivated the application of distributed multi-agent systems (MASs) which could be widely used in power systems for smarter operation and better situational awareness through the utilization of online information and communication technologies [10]. The concept of MASs has gained significant attention in power system protection and security due to their distributed characteristics, dynamic adaptability, and flexibility. An MAS provides a more flexible way of increasing both the resiliency and efficiency through the combination of top-down and bottom-up autonomous decision-making intelligence in a distributed environment. A hierarchical distributed multi-agent framework has the capability of maintaining interactions among different physical operational processes and agent activities through proper communication. The agents in a distributed multi-agent framework use the online information and energy flow to communicate with each other and make a decision which could be used in power systems for the coordination of protection devices to enhance the transient stability.

Although transient stability assessment is an established research area in power systems, there are a few applications of MASs. A conventional time-domain simulation approach in an agent-based framework, for supervision and control to analyze the transient stability of power systems, is proposed in [11]. A real-time closedloop wide-area decentralized power system stabilizers (WDPSSs) based on the reinforcement learning (RL) method and multi-agent framework is discussed in [12]. In [13, 14], a combination of prediction and control agents is used to developed a multi-agent framework where the prediction agents predict the instability within the power system and the control agents stabilize the system based on the information provided by the prediction agents. A multi-agent-based coordinated control scheme is proposed in $[15,16]$ where multi-agent framework is developed through wide area measurements (WAMs). In [17, 18] an output tracking agent is used to track the output where the output of the generator is considered as the rotor angle of the generator and the control agent is used to initiate the turbine valving control. In practice, the rotor angle of generators may not be conveniently measured $[19,20]$. The multi-agent frameworks so far presented in this paper are mainly based on the assumption of a fixed trivial rather than variable CCT. Since the CCT varies with disturbances within the system such as changes in loads and faults within the system, the consideration of varying CCT is of paramount importance for transient stability assessment in a multi-agent environment. Recent works on transient stability improvement in the light of variable CCT consideration using multi-agent scheme can be seen in [21-23].

This paper aims to design a distributed multi-agent architecture in which each intelligent agent represents a specific physical device model with a particular definition to enable information flow among themselves. The proposed MAS helps to dynamically adapt any change in versatile system conditions due to its distributed characteristics. For example, if a fault occurs or the load changes within the physical power system, the MAS adapts these changes to calculate the CCT and assists protection devices by providing a real-time coordination of circuit breaker (CB) operation. Moreover, the implementation of distributed agent-based framework significantly reduces the complexities in power system protection and its relevant computational burdens. In this paper, the CCT is estimated first using the direct method and then determined from the time-domain simulations in order to ensure faster and accurate CCT information. This CCT is computed under a computing facility which has direct access to communicate with the agents related to the protection relays. Since the agents are capable of continuously monitoring the changes in power systems, their dynamic adaptability provides a continual streaming of CCT information to the agents which initiate the operation of CBs. The superiority of the proposed multi-agent scheme is investigated through simulation results under different operating scenarios and it is seen that the proposed scheme enhances the stability limits as compared to the conventional approach $[7,8]$.

The rest of the paper is organized as follows: Section 2 presents the MAS architecture for power systems; individual activity of distributed MAS is presented in Section 3; Section 4 discusses the performance evaluation with illustrative examples and simulation results; and finally, the paper is concluded with brief remarks in Section 5.

\section{Proposed multi-agent framework for power systems}

The MAS is a group of multiple interacting distributed intelligent agents within an environment where they can respond to the events in the surrounding environment with independent control actions. It provides an effective platform for modeling autonomous decision-making entities for power grid operation [24]. An MAS is widely used for advance management of the complex problems, such as control and protection of electrical power networks in a distributed way [25]. In power systems, all physical devices such as generators, transformers, loads, 


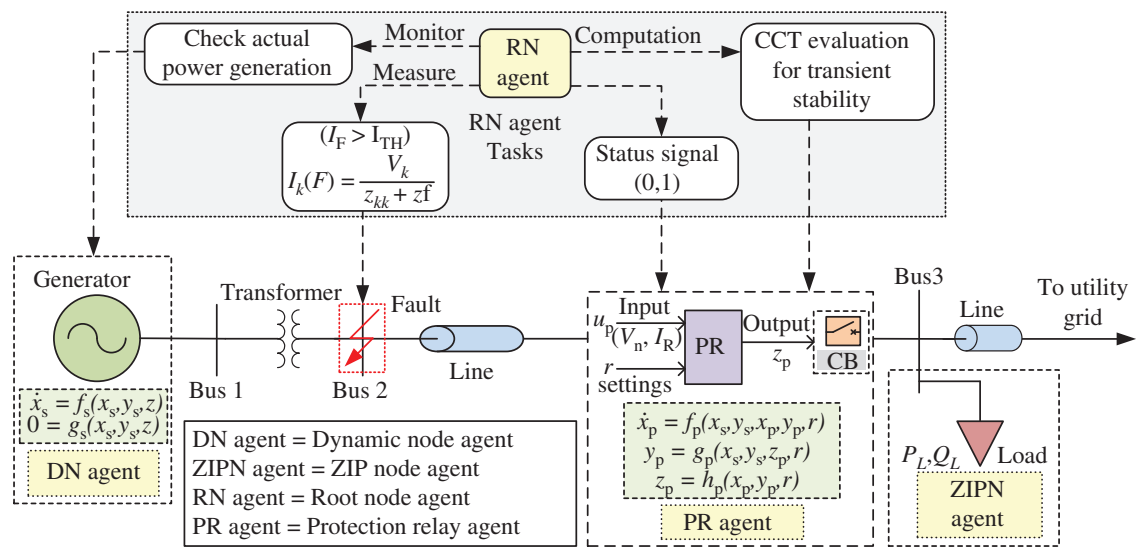

Figure 1: Distributed multi-agent framework for power systems.

protections relays (PRs), and CBs are installed in a distributed manner throughout the whole network.

Phasor measurement units (PMUs) along with the global positioning system (GPS) technology have been currently used in the automation of power systems, especially to provide breaker status signals for proper relay coordinations [26]. Therefore, the advent of PMUs has brought a new dimension to power system automation systems in a multi-agent framework. Figure 1 shows an architecture of a simple power system model where a generator is connected to the utility grid and supplying some loads though a transmission network equipped with CBs and PRs in a multi-agent framework. In this framework, different nodes within the physical model of the power system such as the locations of the generators, PRs with CBs, PMUs and loads have been considered as different agents that have the flexibility to gather and share information from each other. If disturbances occur within the physical power system which is in operation, the MAS is designed in such a way that the agents have the capability to adapt these disturbances to implement their autonomous control actions.

In the proposed multi-agent framework as shown in Figure 1, each agent has been defined to represent particular physical device. The dynamic node (DN) agent comprises the information of the generator connected to the system, whereas the load information has been gathered through ZIP (Z - impedance, I - constant current, $\mathrm{P}$ - constant power) node (ZIPN) agent. The root node (RN) agent comprises PMUs for monitoring, measuring and providing breaker status signal as well as CCT computing facility and lastly, the agent that is embedded within the PR equipments is considered as the PR agent. In this distributed multi-agent framework, the group of node agents associated with the physical device model are considered as an individual subsystem. Each subsystem is connected physically with each other as well as through communication provided by agents. In this paper, it is assumed that the smart power system already has necessary communication network superimposed on its physical layer which is also valid for practical implementation as a practical system has high-speed wireless communication or fiber optic communication facilities. The details of all agents along with their activities have been discussed in the following section with an aim to enhance the transient stability of power systems.

\section{Agent activities for transient stability enhancement}

Since the multi-agent framework is the collection of several intelligent agents, each agent is responsible for performing some specific or a set of specific activities,. In the developed multi-agent framework for power systems, the agent activities have been aimed to achieve a desired goal which is transient stability enhancement of whole power system. The activities of each agent are discussed in the following subsections.

\subsection{Activities of DN agent}

In this paper, DN agent is designed to capture the dynamics of the generator and the generators are considered as synchronous generators. The elements of the system dynamics consists of constrained set of non-linear differential equations in the form (1) using phasor variables [23]: 


$$
\begin{aligned}
& \dot{x}_{\mathrm{s}}=\mathrm{f}_{\mathrm{s}}\left(x_{\mathrm{s}}, y_{\mathrm{s}}, z\right) \\
& 0=\mathrm{g}_{\mathrm{s}}\left(x_{\mathrm{s}}, y_{\mathrm{s}}, z\right)
\end{aligned}
$$

where $x$ is the system state variable $x \in \mathbb{R}^{n}, y$ is the algebraic variable $y \in \mathbb{R}^{m}, z$ is the vector of system parameters $z \in \mathbb{R}^{l}, f$ is the function of the differential equations $f: \in \mathbb{R}^{n} \times \in \mathbb{R}^{m} \times \in \mathbb{R}^{l} \rightarrow \in \mathbb{R}^{n}$ and $g$ is the function of the algebraic equations $g: \in \mathbb{R}^{n} \times \in \mathbb{R}^{m} \times \in \mathbb{R}^{l} \rightarrow \in \mathbb{R}^{m}$.

When a disturbance occurs in the power system, the characteristics of the generator will change depending on the nature of the disturbances. The dynamic models of such generators are essential in order to adapt these changing characteristics with DN agents. However, the actual behaviors of a synchronous generator in real power systems are very complex and difficult to model unless some standard assumptions are considered. It is well known that a third-order synchronous generator model, represented as the voltage behind direct-axis transient reactance, is reliably suitable for transient stability analysis which can be expressed as [20]

$$
\begin{aligned}
\dot{\delta}_{i}= & \omega_{i}-\omega_{0 i} \\
\dot{\omega}_{i}= & -\frac{D_{i}}{2 H_{i}}\left(\omega_{i}-\omega_{0 i}\right) \\
& +\frac{\omega_{0 i}}{2 H_{i}} P_{\mathrm{m} i}-\frac{\omega_{0 i}}{2 H_{i}}\left(E_{\mathrm{q} i}^{\prime 2} G_{i i}+E_{\mathrm{q} i}^{\prime} \sum_{j=1}^{n} E_{\mathrm{q} j}^{\prime} B_{i j} \sin \delta_{i j}\right) \\
\dot{E}_{\mathrm{q} i}^{\prime}= & -\frac{1+\left(x_{\mathrm{d} i}-x_{\mathrm{d} i}^{\prime}\right) B_{i i}}{T_{\mathrm{do} i}} E_{\mathrm{q} i}^{\prime}+\frac{x_{\mathrm{d} i}-x_{\mathrm{d} i}^{\prime}}{T_{\mathrm{do} i}} \sum_{j=1}^{n} E_{\mathrm{q} j}^{\prime} B_{i j} \cos \delta_{i j} \\
& +\frac{1}{T_{\mathrm{do} i}} E_{\mathrm{f} i}
\end{aligned}
$$

where the subscript $i$ represents the synchronous generator connected to ith bus, $\delta$ is the power angle, $\omega$ is the rotor speed, $\omega_{0}$ is the synchronous speed, $H$ is the inertia constant, $P_{\mathrm{m}}$ is the mechanical input power, $D$ is the damping constant, $E_{\mathrm{q}}^{\prime}$ is the quadrature-axis transient voltage, $E_{\mathrm{q}}$ is the quadrature-axis voltage, $T_{\mathrm{do}}$ is the direct-axis opencircuit transient time constant, $E_{\mathrm{f}}$ is the equivalent voltage in the excitation coil, $x_{\mathrm{d}}$ is the direct-axis synchronous reactance, $x_{\mathrm{d}}^{\prime}$ is the direct-axis transient reactance, $G_{i i}$ and $B_{i i}$ are the self-conductance and self-susceptance of $i$ th line, respectively, $G_{i j}$ and $B_{i j}$ are the conductance and susceptance between $i$ th and $j$ th lines, respectively, $\delta_{i}$ is the power angle deviation between $i$ th and $j$ th lines, $I_{\mathrm{d}}$ and $I_{\mathrm{q}}$ are the direct-axis and quadrature-axis currents, respectively.

\subsection{Activities of ZIPN agent}

The ZIPN agent is designed to collect the aggregated load data and share this information with other agents. Since the loads in power systems continuously change, the ZIPN agent is designed to incorporate all variations in load conditions. The static load model is considered to represent this agent which can be described by the following equations [27]:

$$
\begin{aligned}
& P_{\mathrm{L}}=P_{0}\left(\frac{V}{V_{0}}\right)^{\alpha} \\
& Q_{\mathrm{L}}=Q_{0}\left(\frac{V}{V_{0}}\right)^{\beta}
\end{aligned}
$$

where $P_{0}$ and $Q_{0}$ are the real and reactive power consumed at the reference voltage $V_{0}$, respectively. The exponents $\alpha$ and $\beta$ depend on the type of loads, e.g. $\alpha=\beta=0$ for constant power load models, $\alpha=\beta=1$ for constant current load models, and $\alpha=\beta=2$ for constant impedance load models. The real and reactive power characteristics of composite ZIP load models are given by [28, 29]

$$
\begin{aligned}
& P_{\mathrm{L}}=P_{0}\left[a_{\mathrm{p}}\left(\frac{V}{V_{0}}\right)^{2}+b_{\mathrm{p}}\left(\frac{V}{V_{0}}\right)+c_{\mathrm{p}}\right] \\
& Q_{\mathrm{L}}=Q_{0}\left[a_{\mathrm{q}}\left(\frac{V}{V_{0}}\right)^{2}+b_{\mathrm{q}}\left(\frac{V}{V_{0}}\right)+c_{\mathrm{q}}\right]
\end{aligned}
$$

where the coefficients $a_{\mathrm{p}}, b_{\mathrm{p}}, c_{\mathrm{p}}, a_{\mathrm{q}}, b_{\mathrm{q}}$ and $c_{\mathrm{q}}$ are the parameters of the model that define the proportion of each component, respectively.

\subsection{Activities of RN agent}

The RN agent is the heart of the proposed MAS as most of the critical decision-making activities are performed by this agent. The RN agent monitors the overall network condition based on the information obtained from PMUs and process all local information gathered from DN and ZIPN agents to calculate the CCT. Finally, this RN agent shares the decision to the $\mathrm{PR}$ agents by providing the status signals for CB operation. The RN agents mainly perform three major activities-fault detection, dynamic adaptability, and CCT calculation. In the following, these activities are discussed in detail.

\subsubsection{Activity 1: Fault Detection}

In power systems, faults and outages are likely to occur. In such cases, an effective fault detection and diagnosis algorithm is essential as this can improve the transient stability and reliability of power systems. In the proposed distributed multi-agent framework, the $\mathrm{RN}$ agent is used to detect the fault along with its location. The current and 
voltage at the relay location can be obtained using potential and current transformer, respectively, and can be written as [30]:

$$
\begin{gathered}
I_{\mathrm{R}}=\frac{V_{\mathrm{s}}-V_{\mathrm{r}}}{Z_{\mathrm{T}}} \\
V_{\mathrm{R}}=V_{\mathrm{s}}-I_{\mathrm{R}} Z_{\mathrm{s}}
\end{gathered}
$$

where $I_{R}$ is the current across the relay, $V_{R}$ is the voltage at the relay, $V_{\mathrm{S}}$ is the sending end voltage, $V_{\mathrm{r}}$ is the receiving end voltage and $Z_{\mathrm{T}}$ is the total impedance of the system. The fault is detected from the following relationship among fault current, relay current and threshold value of relay current:

$$
I_{\mathrm{f}}= \begin{cases}I_{\mathrm{R}}>I_{\mathrm{TH}} ; & \text { Fault detected } \\ I_{\mathrm{R}}<I_{\mathrm{TH}} ; & \text { No fault }\end{cases}
$$

where $I_{\mathrm{f}}$ is the fault current, $I_{\mathrm{R}}$ is the current flowing through the relay, and $I_{\mathrm{TH}}$ is the threshold of the relay current which is set to each relay for precisely detecting the fault. When a fault occurs within the power system, RN agents measure fault currents and compare these current values with the threshold values of relay currents. If the fault current exceeds the threshold value, the RN agent detects this as a fault and takes necessary steps to trip the CBs through PR agents. Now if there are no faults within the system, the current across the relay is less than the threshold value that ensures the normal operation of power systems. The following relationship has been used in this paper in order to obtain the location of the fault through RN agents [30]:

$$
I_{k}(F)=\frac{V_{k}}{Z_{k k}+Z_{\mathrm{f}}}
$$

where $k$ is the bus number where the fault occurs, $I_{k}$ is the fault current magnitude, $V_{k}$ is the fault bus voltage, $Z_{k k}$ is the element of the bus impedance matrix which is indeed the Thevenin's impedance as viewed from the faulted bus and $Z_{\mathrm{f}}$ is the fault impedance. Using the above expression, the RN agent can determine the bus which is affected by faults.

\subsubsection{Activity 2: Dynamic Adaptability}

The RN agent continuously monitors the load conditions by taking information from DN agents and the same time calculate CCT based on the information gathered from DN agent. For example, if the nominal load $P_{\mathrm{L}}$ changes, the DN agent will adjust the mechanical power input $P_{\mathrm{m}}$ to the generator to pick up the extra loads since stable operation requires a continuous match between the energy input to the prime movers and the new load $P_{\mathrm{L}}^{\prime}$. The RN agent receives this information from DN and ZIPN agents and uses the following logic to adapt to the changes in loads for the new input mechanical power:

$$
\begin{gathered}
P_{\mathrm{m}}=P_{\mathrm{L}} \text { (for normal operation) } \\
\text { if } P_{\mathrm{m}} \gtrless P_{\mathrm{L}}\left(P_{\mathrm{L}} \pm \%\right. \text { load change) } \\
P_{\mathrm{m}}^{\prime}=P_{\mathrm{L}}^{\prime} \text { (for new load) } \\
\text { else } P_{\mathrm{m}}=P_{\mathrm{L}} \text { (for given load) }
\end{gathered}
$$

After gathering the new load information from ZIPN agents and the new mechanical power input information from DN agents, the RN agent calculates the new CCT information which is discussed in the next activity.

\subsubsection{Activity 3: СCT Calculation}

To calculate the CCT, the combination of both direct and time-domain simulation methods is used as each approach has some limitations, as overshadowed earlier in this paper. The expression for the transient energy function for a third-order model can be written as [31, 32]

$$
\begin{aligned}
E_{\mathrm{T}}= & \frac{1}{2} \sum_{i=1}^{n} M_{i} \omega_{i}^{2}-\sum_{i=1}^{n} \int_{\delta_{i}}^{\delta_{i}^{\mathrm{s}}} f_{i}\left(\delta_{i}\right) d \delta_{i}+\frac{x_{\mathrm{d}} \sum}{2\left(x_{\mathrm{d}}-x_{\mathrm{d}}^{\prime}\right)} \frac{1}{x_{\mathrm{d}}^{\prime} \sum}\left[E_{\mathrm{q}}^{\prime}\right]^{2} \\
= & \frac{1}{2} \sum_{i=1}^{n} M_{i} \omega_{i}^{2}-\left(\sum_{i=1}^{n} P_{i}\left(\delta_{i}-\delta_{i}^{\mathrm{s}}\right)\right. \\
& \left.+\sum_{i=1}^{n} E_{\mathrm{q} i}^{\prime} \sum_{j=1}^{n} E_{\mathrm{q} j}^{\prime} B_{i j} \cos \left(\delta_{i j}-\delta_{i j}^{\mathrm{s}}\right)\right)+\frac{x_{d} \sum}{2\left(x_{\mathrm{d}}-x_{\mathrm{d}}^{\prime}\right)} \frac{1}{x_{\mathrm{d}}^{\prime}} \sum\left[E_{\mathrm{q}}^{\prime}\right]^{2}
\end{aligned}
$$

where $M_{\mathrm{i}}=\frac{2 H_{\mathrm{i}}}{\omega_{\mathrm{s}}}, \quad P_{i}=P_{\mathrm{m} i}-E_{\mathrm{q} i}^{\prime 2} G_{i i}, \quad \delta_{i}^{\mathrm{s}}$ is the post-fault stable equilibrium point (s.e.p) which can be written as $\delta_{i}^{s}=\sin ^{-1}\left(\frac{P_{\operatorname{mi}}}{P_{\mathrm{ei}} \mathrm{m}}\right)$. The first part of the right-hand side of eq. (8) is the transient kinetic energy $\left(E_{\mathrm{KE}}\right)$, second part is the potential energy $\left(E_{\mathrm{PE}}\right)$ of the system and lastly, the third part is the energy $\left(E_{E_{\mathrm{q}}^{\prime}}\right)$ due to quadrature-axis transient voltage of the synchronous generator.

In this paper, the CCT is first estimated by the direct method using the potential energy boundary surface (PEBS) approach, which was first introduced in [33, 34]. It has received wide attention from researchers as it avoids computing the controlling unstable equilibrium points $\left(\delta_{i}^{\mathrm{u}}=\pi-\delta_{i}^{\mathrm{s}}\right)$ [31, 35]. The critical energy $\left(E_{\mathrm{cr}}\right)$ can be obtained from the PEBS, the crossing of which is the point at which the function, $f_{i}\left(\delta_{i}\right) \cdot\left(\delta_{i}-\delta_{i}^{s}\right)=f_{i}\left(\delta_{i}\right) \cdot \hat{\delta}_{i}=0$, and is 
approximately the same point at which $E_{\mathrm{PE}}$ is maximum along the faulted trajectory. The following steps are used to calculate the CCT.

1. Perform a load flow analysis to obtain the initial, pre and post-fault conditions.

2. During the fault, solve eq. (1) to obtain the state variables. Also compute the post-fault s.e.ps and the fault on trajectory.

3. Observe the potential energy $\left(E_{\mathrm{PE}}\right)$ at each time step integration, where its parameters pertain to the postfault configuration.

4. Continue steps 2 and 3 until the function $f_{i}\left(\delta_{i}\right) \cdot \hat{\delta}_{i}=0$. This is the PEBS crossing at which point the maximum value of $E_{\mathrm{PE}}$ is obtained and is a good estimation of $E_{\text {cr }}$ for the applied fault.

5. Find when the total energy reaches the same value as critical energy, i.e. $E_{\mathrm{T}}=E_{\mathrm{cr}}$ and this instant of time while $E_{\mathrm{T}}$ crosses the maximum value of $E_{\mathrm{PE}}$ or $E_{\mathrm{cr}}$ is the estimated CCT $\left(t_{\mathrm{cr}}\right)$ of the system.

6. The fault start and end times, estimated minimum $\left(t_{\mathrm{cr}}^{\min }\right)$ and maximum $\left(t_{\mathrm{cr}}^{\max }\right)$ fault clearing times, and time-domain simulation step-size $(D)$ are set up for the time-domain approach.

7. A load flow analysis is conducted and the transient stability obtained from the time-domain simulation method is evaluated using the dynamic equations.

8. The minimum interval of the CCT is searched and then the stability is checked based on the simulation results.

9. The CCT calculation is successful when the system is stable for the minimum fault clearing time and unstable for the maximum.

The flowchart of the whole CCT calculation process is shown in Figure 2. With the calculated CCT, the RN agent communicates with PR agents to share this information for proper relay coordination.

\subsection{Activities of PR agent}

The PR agents are designed to capture a set of protection parameters and the equations related to the PR agents are given as follows [36]:

$$
\begin{aligned}
\dot{x}_{\mathrm{p}} & =\mathrm{f}_{\mathrm{p}}\left(x_{\mathrm{s}}, y_{\mathrm{s}}, x_{\mathrm{p}}, y_{\mathrm{p}}, r\right) \\
y_{\mathrm{p}} & =\mathrm{g}_{\mathrm{p}}\left(x_{\mathrm{s}}, y_{\mathrm{s}}, x_{\mathrm{p}}, r\right) \\
z_{\mathrm{p}} & =\mathrm{h}_{\mathrm{p}}\left(x_{\mathrm{p}}, y_{\mathrm{p}}, r\right)
\end{aligned}
$$

where $x_{\mathrm{p}}$ and $y_{\mathrm{p}}$ are the internal dynamics of the PR and CB which follow a set of logic signals depend on the local state variables, e.g. node voltage and current through PR, $r$ is the

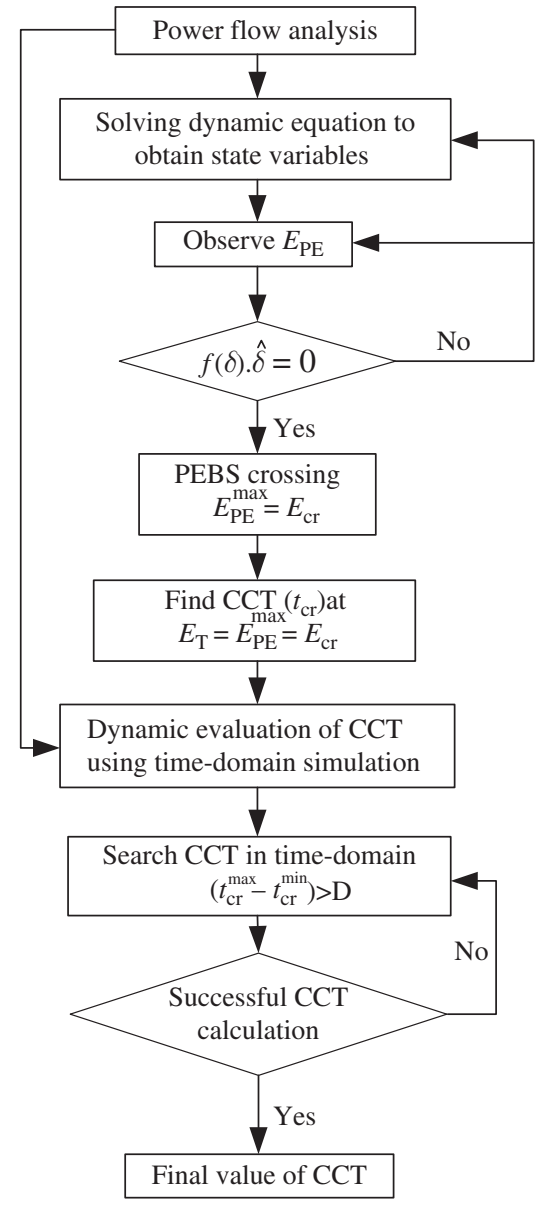

Figure 2: Flowchart of the CCT calculation.

relay settings under various operating conditions, $z_{\mathrm{p}}$ is the output signal of PRs act as input to the CBs for their operation. The functions $f_{\mathrm{p}}, g_{\mathrm{p}}$ and $h_{p}$ are discontinuous as they provide $\mathrm{CB}$ operation at any instant of time. Usually, $u_{\mathrm{p}}$, i.e. the node voltage and the current across the relay are the input to the PR. The output, $z_{\mathrm{p}}$, of the PR is the tripping logic signal fed to the $\mathrm{CB}$ for opening and closing its contact.

The PR agents process the CCT information through PRs to initiate the breaker operation. PR agents continuously receive updated CCT information based on the changes within the power system adapted through RN agent and act accordingly. PR agents use a simple control logic for the operation of $\mathrm{CBs}$ as well as to provide $\mathrm{CB}$ status signals. The control logic with status signals for opening and closing CBs can be written as:

$z_{\mathrm{p}}=C_{i}(t)= \begin{cases}0, & \text { for opening of CB at time } t \\ 1, & \text { for closing of CB at time } t\end{cases}$

where $C_{i}$ is the breaker signal for $i$ th breaker and $i=1,2,3, \ldots, n$. Under normal operating conditions, i.e. where there are no faults within the system the breaker signal status is 1 . However, PR agents continuously 
collect the updated CCT information from RN agents if any disturbance occurs in power systems. PR agents take necessary action for the operation of CBs based on the collected CCT information from RN agents and share their activities with other agents. In practice, IEC61850 protocol is used to communicate status and commands between the relays within the substations in which IEC61850 GOOSE messages utilize multi-cast Ethernet to provide a real-time support for protection mechanisms and used for communication among relay equipments [37]. However, failures in communication or added complexity of the system due to latency of the CB operation may cause malfunctioning of protection devices. A recent work on the detection and identification of erroneous data in the MAS-based protection framework can be seen in [38]. In order to provide a real-time coordination of protection devices, equivalent PR agents have also been developed in Java Agent Development Framework (JADE) platform.

Finally, the agent activities have been summarized through a flowchart as shown in Figure 3 from where it can be seen that each agent can access to the power system model and communicate with each other for initiating the PR coordination. The transient stability enhancement of power systems, with the proposed distributed multi-agent scheme, is discussed in the following section.

\section{Performance evaluation of distributed MAS}

The IEEE 39-bus New England test system [39] as shown in Figure 4 is considered to evaluate the effectiveness of

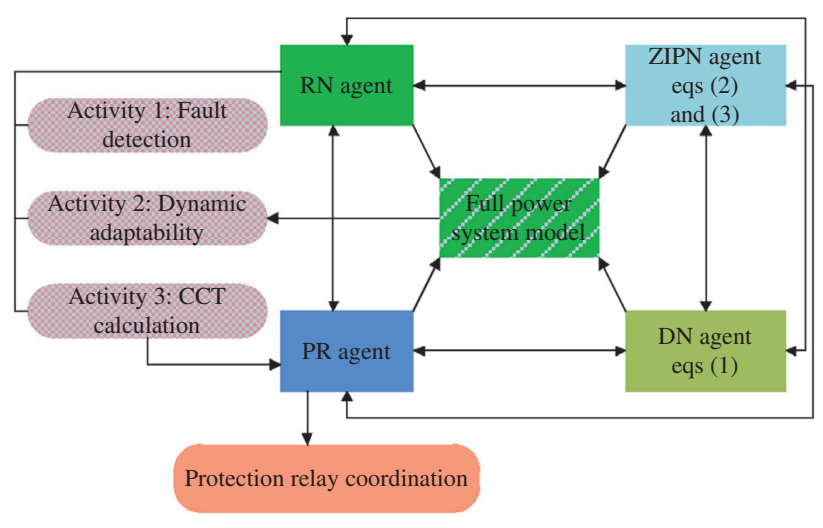

Figure 3: Flowchart of agent activities. the proposed distributed MAS for enhancing the transient stability of a large-scale power system. This system comprises 39 buses, with 10 generators and 19 loads totaling 6,150.1 MW and 1,233.9 MVAr of real and reactive powers, respectively. Each generator is modeled as a third-order synchronous generator with an IEEE type 2 exciter, except that at bus-31 which is arbitrarily considered a slack bus, and has a simple turbine governor, except generator 1. For simplicity, the whole system is divided into four zones, each of which provides independent CB control operations regarding the buses.

The zoning of IEEE 39-bus system can be seen in Figure 4 and summarized as follows:

- Zone 1: generators at bus-30 and bus-39, and loads at bus-3, bus- 4 , bus-7, bus- 8 and bus-39.

- Zone 2: generators at bus-31 and bus-32, and loads at bus-11, bus-15 and bus-31.

- Zone 3: generators at bus-33, bus-34, bus-35 and bus36 , and loads at bus- 16 , bus- 20 , bus- 21 , bus- -23 and bus-24.

- Zone 4: generators at bus-37 and bus-38, and loads at bus-18, bus-25, bus-26, bus-27, bus-28 and bus-29.

These zones are interconnected through seven lines: Zone 1 and Zone 2 through lines L- 8 from bus- 4 to bus-14, L-10 from bus- 5 to bus- 6 and L-11 from bus- 7 to bus-31; Zone 2 and Zone 3 through line L-20 from bus- 15 to bus16; Zone 3 and Zone 4 through line L-21 from bus-16 to bus-17; and Zone 1 and Zone 4 through lines L-4 from bus- 2 to bus- 25 and L- 6 from bus- 3 to bus- 18 .

In this paper, generators at bus-34 (G4) and bus-38 (G9) are considered to compare the results since they have the highest participation in the system instability as the eigenvalues of dominant unstable mode are $0.35031 \pm 2.8725 i$ [39]. Since the CCT is susceptible to changes in power system configurations, the performances of the developed MAS for different ones are demonstrated. In this paper, the following two cases: (i) short-circuit faults within the network, and (ii) the combination of short-circuit faults and generator load variations; have considered to demonstrate the transient stability of power systems in a multi-agent framework along with a comparison to a conventional approach.

- Case 1: Performance evaluation under three-phase short-circuit faults

During the normal operation of a power system, the agents within the system do not take any action rather than continuous monitoring activities. The agent activities start when the RN agent detects a fault. In this simulation, a three-phase short-circuit fault, which is 


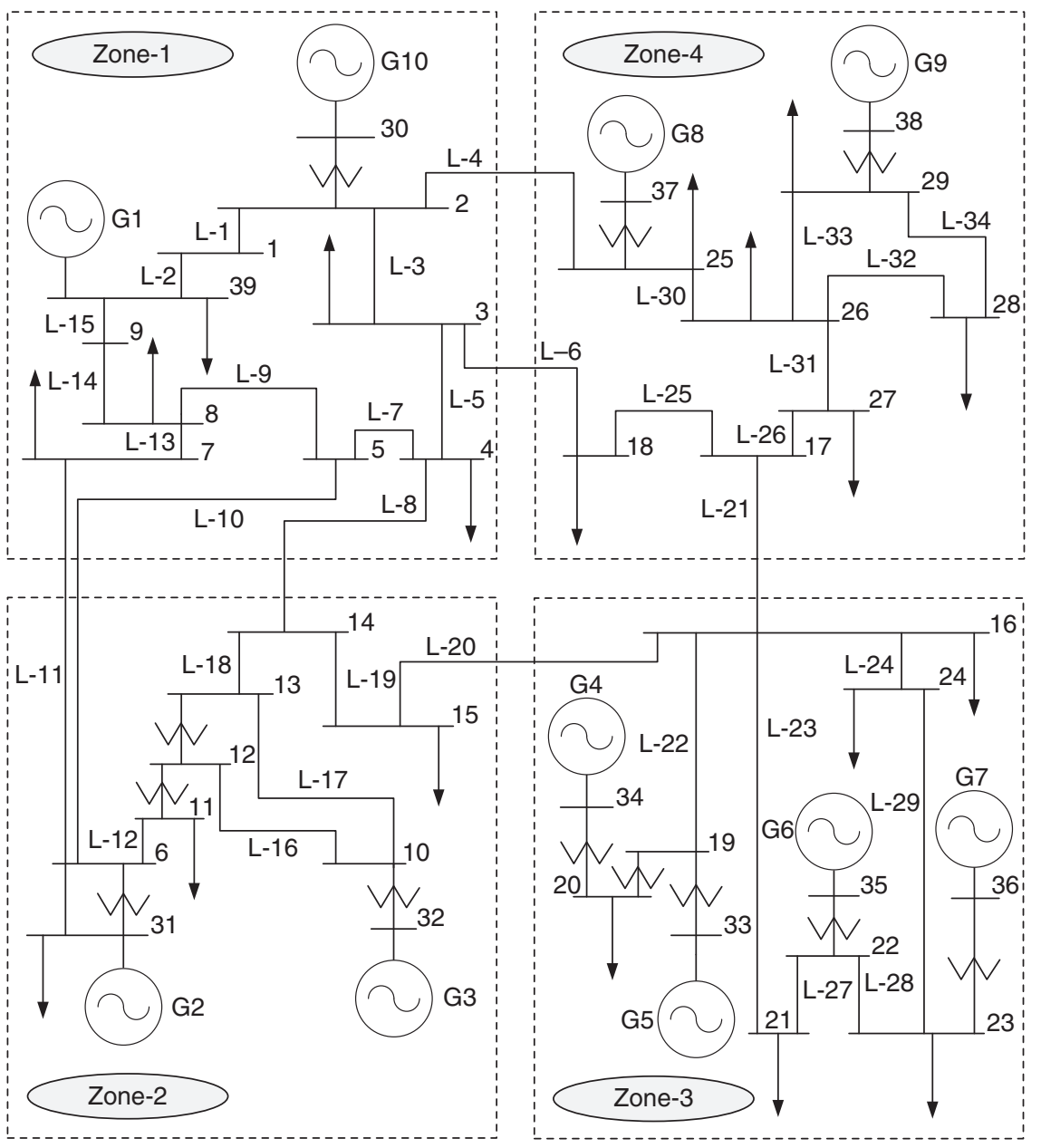

Figure 4: Single-line diagram of IEEE 39-bus New England test system.

the most severe fault in power systems, is applied to evaluate the performance of the designed scheme. This fault is applied at bus-16 and the following fault sequence is considered.

- Fault occurs at $t=1 \mathrm{~s}$

- Fault is cleared at $t=1.2 \mathrm{~s}$

When the fault is applied at bus-16, the behavior of the current is changed across the relay, i.e. suddenly increases. Since a predefined threshold value of fault current is set in each relay protecting the corresponding line, the RN agent detects that fault while its current flows above the threshold value as shown in Figure 5. The generators are still supplying the nominal loads, i.e. the loads within the system remain unchanged. The ZIPN and DN agents send their information to the $\mathrm{RN}$ agent which is required to detect the fault, calculate the CCT and share this information with the PR agent which initiates the operation of CBs with a status signal, $C_{i}=0$, to isolate the faulty part from the rest

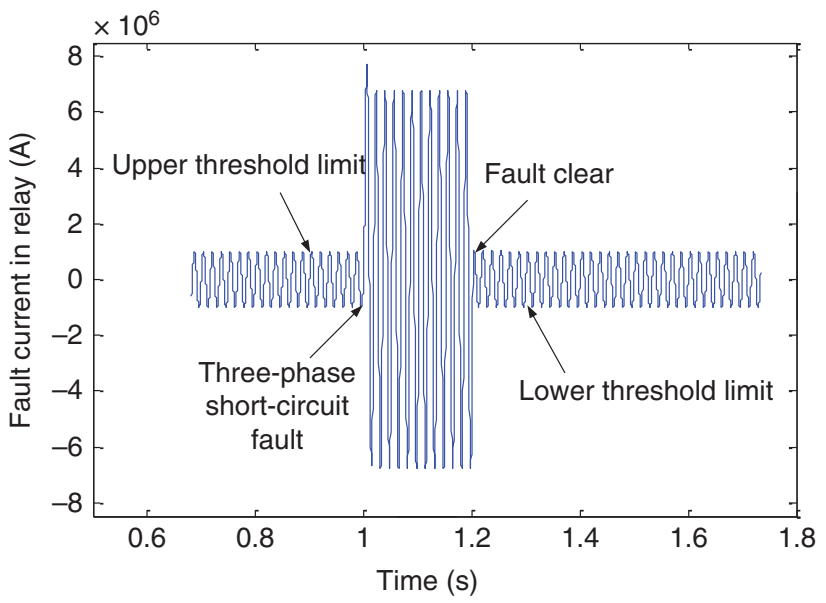

Figure 5: Current across the relay when fault occurs at bus-16.

of the system. In this case, the transmission line connected between bus-15 and bus-16, i.e. line 15-16 is tripped. For the applied three-phase short-circuit fault at bus-16, the 


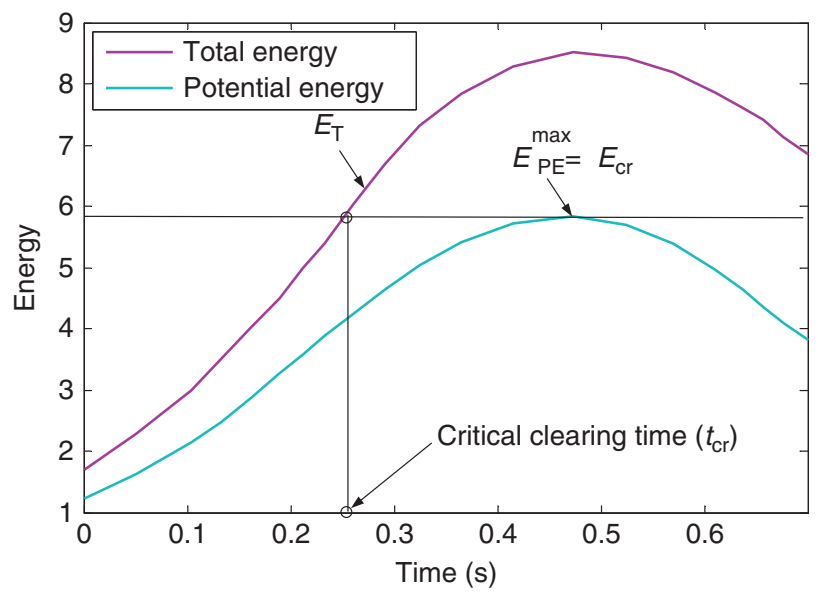

Figure 6: Total energy and potential energy when fault occurs at bus-16.

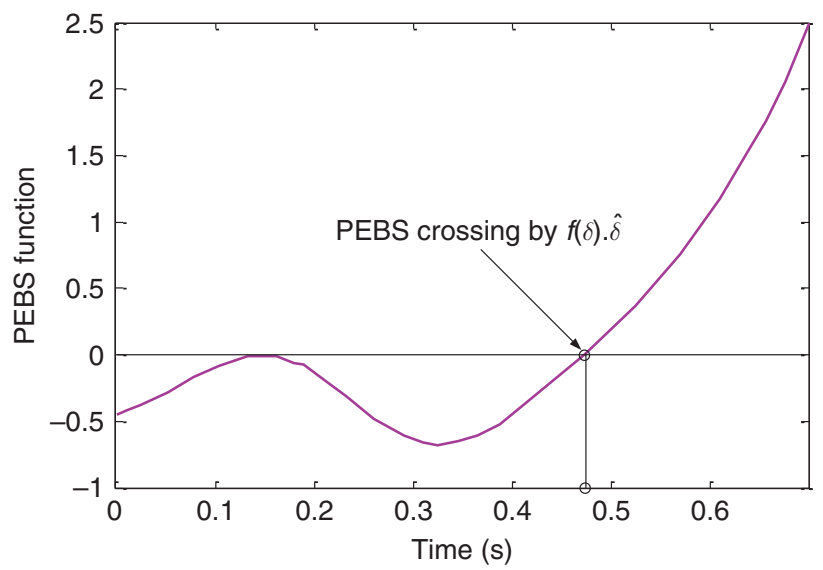

Figure 7: PEBS crossing by function $f(\delta) . \hat{\delta}$.

response of the total energy and the potential energy is shown in Figure 6, in which it can be seen that $E_{\mathrm{PE}}^{\max }$ is reached at $0.473 \mathrm{~s}$ which is the critical energy $\left(E_{\mathrm{cr}}\right)$ of the system. Also, the zero crossing of the function, $f_{i}\left(\delta_{i}\right) . \hat{\delta}_{i}$, occurs at approximately the same time as seen from Figure 7.

In Figure 6, it is seen that the graph of total energy $\left(E_{\mathrm{T}}\right)$ crosses $E_{\mathrm{PE}}^{\max }$ or $E_{\mathrm{cr}}$ at $t=t_{\mathrm{cr}}=258 \mathrm{~ms}$, which is the CCT of the system. This CCT is used to estimate the original CCT calculation through the time-domain approach which is found by the RN agent to be $264 \mathrm{~ms}$. At the instant of transmission line isolation; an inter-agent communication takes place to confirm operation of the PR agents with the calculated CCT information with a communication delay of 0.3 cycle added to the CCT information, i.e. $269 \mathrm{~ms}$ as final value of CCT shown in Table 1. When the fault is cleared, the RN agent shares this information with the PR agents which initiate a reverse action, i.e. generates a status signal, $C_{i}=1$, in order to reclose the CBs. At this stage, the transmission line $15-16$ is reconnected to the system and the system resumes its normal operation.

Now, if the system is simulated with a conventional time-domain approach for the CCT calculation and a threephase short-circuit fault with the aforementioned fault sequence is applied at bus-16, the CCT is $241 \mathrm{~ms}$ which is also shown in Table 1 . The CCT from the time-domain simulation is less than that from the proposed scheme due to the lack of information being exchanged among the different subsystems. In this case, the transient stability limit of the system is also less as the maximum time during which a fault can be applied is less as compared to the designed distributed multi-agent framework. The proposed scheme enhances the transient stability limit by $12.05 \%$ as shown in Table 1 when a fault is applied at bus-16. The corresponding rotor angles of the synchronous generators (G4 and G9) are shown in Figures 8 and 9 for both conventional (blue line) and proposed (green line) approaches from which it can be seen that the rotor angle oscillations are less with the developed multi-agent scheme. Figures 10 and 11 show the subsequent generator rotor speeds in which it can be seen that their oscillations are less with the proposed scheme and they return to their original synchronous value, i.e. $1 \mathrm{pu}$, after successful relay coordination by agents.

\section{- Case 2: Performance evaluation under three-phase short-circuit faults and generator load changes}

In this case study, the performance of the designed scheme is evaluated by considering a different scenario, a combination of a three-phase short-circuit fault and changes in generator loads. The fault is applied at bus16 and the similar fault sequence, i.e. fault occurring at $t=1 \mathrm{~s}$ and clearing at $t=1.2 \mathrm{~s}$, is considered. The loads

Table 1: Comparison of transient stability limits for three-phase short-circuit fault.

\begin{tabular}{|c|c|c|c|c|c|}
\hline \multirow{2}{*}{$\begin{array}{l}\text { Fault } \\
\text { Location }\end{array}$} & \multirow{2}{*}{$\begin{array}{l}\text { Tripped } \\
\text { Line }\end{array}$} & \multicolumn{3}{|c|}{ Critical clearing time (CCT) information (s) } & \multirow{2}{*}{$\begin{array}{r}\text { Transient stability } \\
\text { Limit improvement (\%) }\end{array}$} \\
\hline & & Conventional & PEBS & Proposed & \\
\hline Bus-16 & Line $15-16$ & 0.241 & 0.258 & 0.269 & 12.05 \\
\hline
\end{tabular}




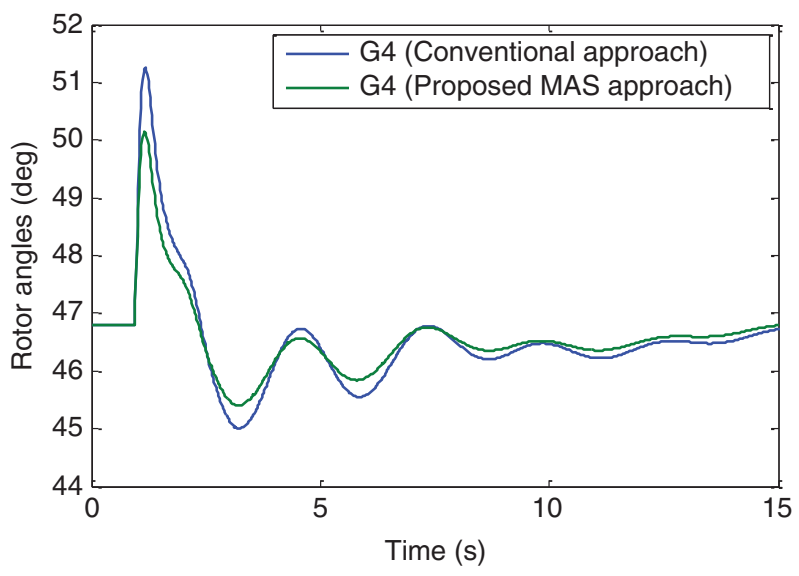

Figure 8: Rotor angle of $\mathrm{G} 4$ when a fault occurs at bus-16 (green line, proposed MAS approach; blue line, conventional approach).

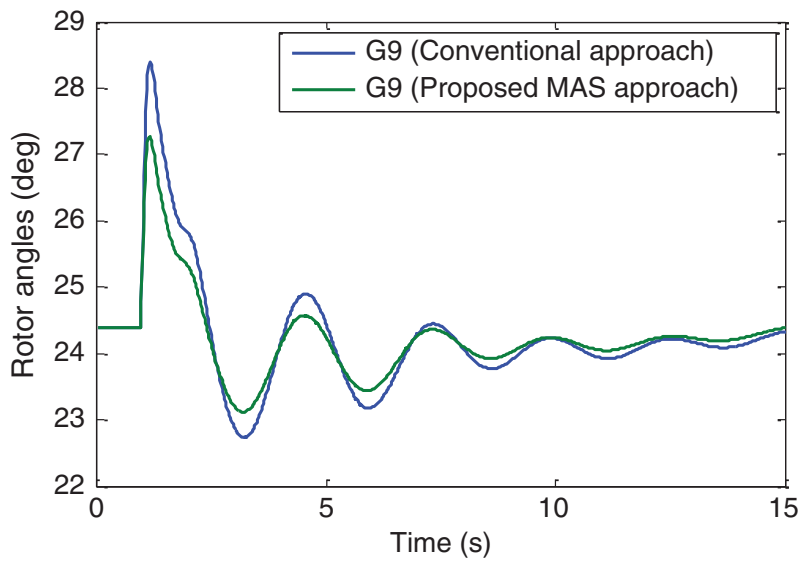

Figure 9: Rotor angle of G9 when a fault occurs at bus-16 (green line, proposed MAS approach; blue line, conventional approach).

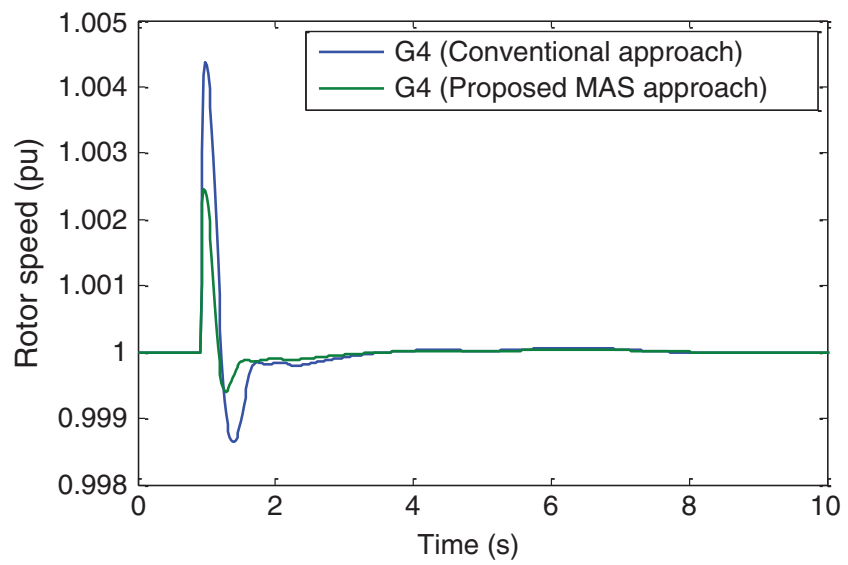

Figure 10: Rotor speed of G4 when a fault occurs at bus-16 (green line, proposed MAS approach; blue line, conventional approach).

of generators G4 and G9 are increased to 5\% from their nominal values to meet the demand requirement assuming that the system load increases. Due to these load

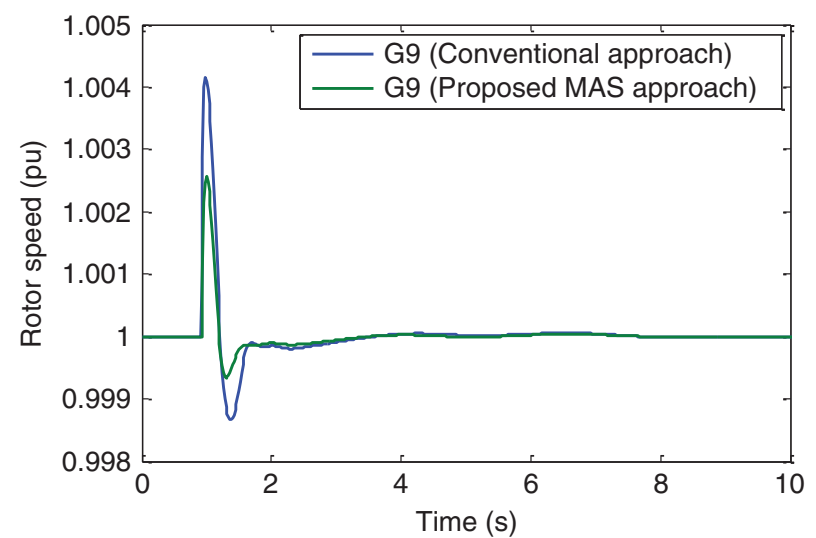

Figure 11: Rotor speed of G9 when a fault occurs at bus-16 (green line, proposed MAS approach; blue line, conventional approach).

changes, the CCT will be different from the previous case, with the new ones shown in Table 2 for both the conventional and proposed approaches. In this scenario, the agents communicate with each other to adapt the changes through the RN agents and calculate the new CCTs in a very similar way to that in the previous case study. The PR agents initiate the breaker status signal, $C_{i}=0$, at the instance of the fault and another status signal, $C_{i}=1$, as soon as the fault is cleared. From this case study, it can be seen that the CCT is more with the proposed approach which can also be seen from Table 2 .

During the faulted condition, the transmission line 15-16 is tripped and reconnected after the clearance of fault by agents. Finally, inter-agent communication takes place to update the final status of each agent. In this case, the transient stability limit is enhanced by $11.46 \%$ as shown in Table 2 using the proposed scheme compared with that of the conventional time-domain simulation method. Also, it can be seen that the CCT decreases with the increase in generator loads which means that the transient stability limit of the system is reduced. Further validation of this point is evident from the rotor angle responses of G4 and G9 as shown in Figures 12 and 13 , respectively.

From both case studies, it is obvious that the designed distributed MAS enhances the transient stability of power systems in a more superior and smarter way than the conventional method.

\section{Conclusion}

A hierarchical distributed multi-agent infrastructure, in which an individual agent provides a powerful framework through representing physical device models in 
Table 2: Comparison of transient stability limits for three-phase short-circuit fault and $5 \%$ changes in generators loads.

\begin{tabular}{|c|c|c|c|c|c|}
\hline \multirow{2}{*}{$\begin{array}{l}\text { Fault } \\
\text { Location }\end{array}$} & \multirow{2}{*}{$\begin{array}{l}\text { Tripped } \\
\text { Line }\end{array}$} & \multicolumn{3}{|c|}{ Critical clearing time (CCT) information (s) } & \multirow{2}{*}{$\begin{array}{r}\text { Transient stability } \\
\text { Limit improvement (\%) }\end{array}$} \\
\hline & & Conventional & PEBS & Proposed & \\
\hline Bus-16 & Line $15-16$ & 0.218 & 0.232 & 0.243 & 11.46 \\
\hline
\end{tabular}

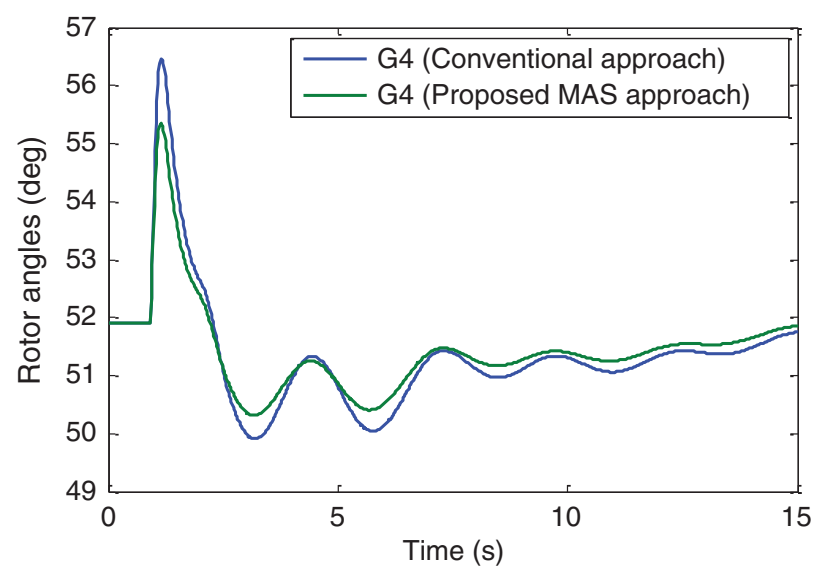

Figure 12: Rotor angle of G4 for fault occurs at bus-16 with $5 \%$ load change (green line, proposed MAS approach; blue line, conventional approach).

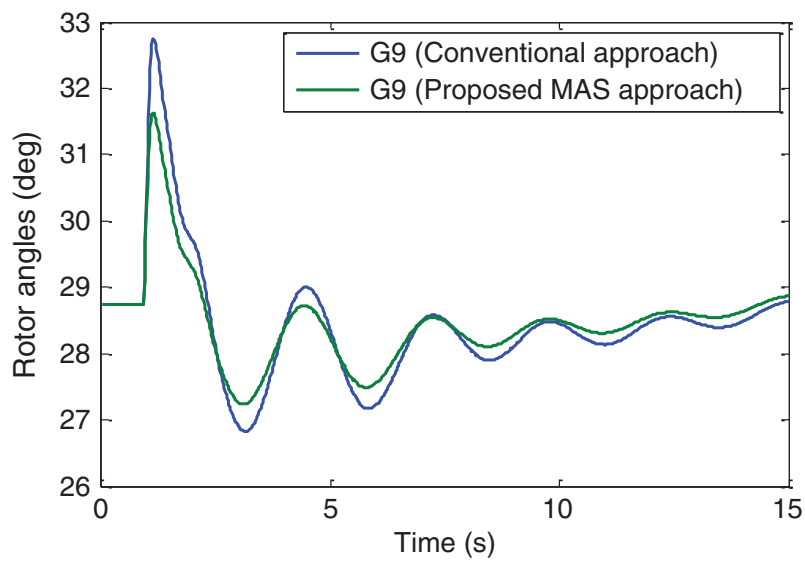

Figure 13: Rotor angle of G9 for fault occurs at bus-16 with $5 \%$ load change (green line, proposed MAS approach; blue line, conventional approach).

power systems to enhance the transient stability, is developed. The agents cooperate and communicate with each other in a distributed fashion to analyze and monitor the condition of the power system. In the designed framework, multiple agents communicate with each other to adapt to changes and maintain a stable operation of a power system by tripping CBs through proper relay coordination based on the continually updated CCT information. Simulation results clearly show that the proposed MAS provides a robust environment for faster and accurate transient stability assessment and a wider stability margin for a large-scale power system as compared to a static CB operation scheme. Future work will deal with the incorporation of control agents within the proposed multi-agent framework for increasing the stability margin of power systems.

\section{Appendix}

Table 3: Machine bus data of IEEE 39-bus New England power system.

\begin{tabular}{lrr}
\hline Machine & Voltage $(\mathbf{p u})$ & Power generation $(\mathbf{M W})$ \\
\hline 1 & 1.04550 & 250.00 \\
2 & 1.04360 & 572.93 \\
3 & 1.02038 & 650.00 \\
4 & 0.99780 & 632.00 \\
5 & 0.99355 & 508.00 \\
6 & 0.99651 & 650.00 \\
7 & 0.94083 & 560.00 \\
8 & 0.95460 & 540.00 \\
9 & 1.01114 & 830.00 \\
10 & 1.00861 & 1005.729 \\
\hline
\end{tabular}

Table 4: Load bus data of IEEE 39-bus New England power system.

\begin{tabular}{lrr}
\hline Bus number & Real load (MW) & Reactive load (MVAr) \\
\hline 3 & 322 & 2.4 \\
4 & 500 & 1.03 \\
7 & 233.8 & 840 \\
8 & 522 & 176 \\
12 & 8.5 & 88 \\
15 & 30 & 153 \\
16 & 329.4 & 323 \\
18 & 158 & 30 \\
20 & 680 & 103 \\
21 & 680 & 103 \\
23 & 247 & 84 \\
24 & 308.6 & -92.2 \\
25 & 224 & 47.2 \\
26 & 139 & 17 \\
27 & 281 & 75.5 \\
28 & 206 & 27.6 \\
29 & 283.5 & 126.9 \\
31 & 9.2 & 4.6 \\
39 & 1104 & 250 \\
\hline
\end{tabular}


Table 5: Dynamic system data of IEEE 39-bus New England power system.

\begin{tabular}{|c|c|c|c|c|c|c|c|c|c|c|}
\hline Machine & 1 & 2 & 3 & 4 & 5 & 6 & 7 & 8 & 9 & 10 \\
\hline$X_{\mathrm{Is}}(\mathrm{pu})$ & 0.0125 & 0.035 & 0.0304 & 0.0295 & 0.027 & 0.0224 & 0.0322 & 0.0028 & 0.00298 & 0.0199 \\
\hline$R_{\mathrm{s}}(\mathrm{pu})$ & 0 & 0 & 0 & 0 & 0 & 0 & 0 & 0 & 0 & 0 \\
\hline$X_{\mathrm{d}}(\mathrm{pu})$ & 0.1 & 0.295 & 0.2495 & 0.262 & 0.33 & 0.254 & 0.295 & 0.29 & 0.2106 & 0.169 \\
\hline$X_{\mathrm{d}}^{\prime}(\mathrm{pu})$ & 0.031 & 0.0697 & 0.0531 & 0.0436 & 0.066 & 0.05 & 0.049 & 0.057 & 0.057 & 0.0457 \\
\hline$X_{\mathrm{d}}^{\prime \prime}(\mathrm{pu})$ & 0.025 & 0.05 & 0.045 & 0.035 & 0.05 & 0.04 & 0.04 & 0.045 & 0.045 & 0.04 \\
\hline$T_{\mathrm{do}}^{\prime}(\mathrm{s})$ & 10.2 & 6.56 & 5.7 & 5.69 & 5.4 & 7.3 & 5.66 & 6.7 & 4.79 & 9.37 \\
\hline$T_{\mathrm{do}}^{\prime \prime}(\mathrm{s})$ & 0.05 & 0.05 & 0.05 & 0.05 & 0.05 & 0.05 & 0.05 & 0.05 & 0.05 & 0.05 \\
\hline$X_{\mathrm{q}}(\mathrm{pu})$ & 0.069 & 0.282 & 0.237 & 0.258 & 0.31 & 0.241 & 0.292 & 0.280 & 0.205 & 0.115 \\
\hline$X_{\mathrm{q}}^{\prime}(\mathrm{pu})$ & 0.028 & 0.060 & 0.050 & 0.040 & 0.060 & 0.045 & 0.045 & 0.050 & 0.050 & 0.045 \\
\hline$X_{q}^{\prime \prime}(\mathrm{pu})$ & 0.025 & 0.05 & 0.045 & 0.035 & 0.05 & 0.04 & 0.04 & 0.045 & 0.045 & 0.04 \\
\hline$T_{\mathrm{qo}}^{\prime}(\mathrm{s})$ & 1.5 & 1.5 & 1.5 & 1.5 & 0.44 & 0.4 & 1.5 & 0.41 & 1.96 & 1.5 \\
\hline$T_{\mathrm{qo}}^{\prime \prime}(\mathrm{s})$ & 0.035 & 0.035 & 0.035 & 0.035 & 0.035 & 0.035 & 0.035 & 0.035 & 0.035 & 0.035 \\
\hline$H(\mathrm{~s})$ & 42 & 30.2 & 35.8 & 28.6 & 26 & 34.8 & 26.4 & 24.3 & 34.5 & 31 \\
\hline$D$ & 4 & 9.75 & 10 & 10 & 3 & 10 & 8 & 9 & 14 & 5.56 \\
\hline
\end{tabular}

Table 6: DC excitation system data of IEEE 39-bus New England power system.

\begin{tabular}{lccccccccc}
\hline Machine & $\boldsymbol{T}_{\mathrm{r}}(\mathbf{s})$ & $\boldsymbol{K}_{\mathrm{A}}$ & $\boldsymbol{T}_{\mathrm{A}}(\mathbf{s})$ & $\boldsymbol{V}_{\max }(\mathrm{pu})$ & $\boldsymbol{V}_{\min }(\mathrm{pu})$ & $\boldsymbol{K}_{\mathrm{E}}(\mathbf{s})$ & $\boldsymbol{T}_{\mathrm{E}}$ & $\boldsymbol{A}_{\mathrm{ex}}$ & $\boldsymbol{B}_{\mathrm{ex}}$ \\
\hline 1 & 0.01 & 40 & 0.02 & 10 & -10 & 1 & 0.785 & 0.07 \\
2 & 0.01 & 40 & 0.02 & 10 & -10 & 1 & 0.785 & 0.07 & 0.91 \\
3 & 0.01 & 40 & 0.02 & 10 & -10 & 1 & 0.785 & 0.07 & 0.91 \\
4 & 0.01 & 40 & 0.02 & 10 & -10 & 1 & 0.785 & 0.07 & 0.91 \\
5 & 0.01 & 40 & 0.02 & 10 & -10 & 1 & 0.785 & 0.07 & 0.91 \\
6 & 0.01 & 40 & 0.02 & 10 & -10 & 1 & 0.785 & 0.07 & 0.91 \\
7 & 0.01 & 40 & 0.02 & 10 & -10 & 1 & 0.785 & 0.07 & 0.91 \\
8 & 0.01 & 40 & 0.02 & 10 & -10 & 1 & 0.785 & 0.07 & 0.91 \\
9 & 0.01 & 40 & 0.02 & 10 & -10 & 1 & 0.785 & 0.07 & 0.91 \\
10 & 0.01 & 40 & 0.02 & 10 & -10 & 1 & 0.785 & 0.07 & 0.91 \\
\hline
\end{tabular}

\section{References}

1. Erlich I, Gessesse B, Shewarega F. Modelling and performance analysis of wind farms: a case study of the ashegoda wind farms in Ethiopia. In: IEEE Power Africa Conf, 2012:1-7.

2. Pota HR, Dong Y. Recent development in direct assessment of transient stability limit. In: Electr Energy Conf, 1990.

3. Kumkratug P. Investigation of the critical clearing time of power system with synchronous machine model including saliency. Am J Appl Sci 2012;9:227-30.

4. Maria G, Tang C, Kim J. Hybrid transient stability analysis. IEEE Trans Power Syst 1990;5:384-93.

5. Chang H, Liu W, Xing Z. Online application of power system transient stability computation. Power Syst Technol 2007;31:54-8.

6. Ren L, Tian F, Yan J, Yu Z, Su F, Wu T. Online application and fast solving method for critical clearing time of three-phase short circuit in power system. Int J Smart Grid Clean Energy 2013;2:93-9.

7. Xue A, Shen C, Mei S, Ni Y, Wu F, Lu Q. A new transient stability index of power systems based on theory of stability region and its applications. In: IEEE Power Energy Soc Gen Meet, 2006.
8. Dinh HN, Nguyen MY, Yoon YT. Novel techniques for real time computing critical clearing time SIME-B and CCS-B. J Electr Eng Technol 2013;8:197-205.

9. Lim I-H, Sidhu T, Choi M-S, Lee S-J, Hong S, Lim S-I, et al. Design and implementation of multiagent-based distributed restoration system in DAS. IEEE Trans Power Delivery 2013;28:585-93.

10. Nguyen C, Flueck A. Agent based restoration with distributed energy storage support in smart grids. IEEE Trans Smart Grid 2012;3:1029-38.

11. Benseddik A, Hasni M, Menaa M, Boussahoua B, Gueddouche $R$, Boudour M. Application of multi-agents system in multimachine power system transient stability and control. In: 3rd Int Conf Syst Contr (ICSC), 2013:1043-48.

12. Hadidi R, Jeyasurya B. A real-time multiagent wide-area stabilizing control framework for power system transient stability enhancement. In: IEEE Power Energy Soc Gen Meet, 2011:1-8.

13. Mohamed MA, Karady GG, Yousuf AM. New strategy agents to improve power system transient stability. World Acad Sci Eng Technol 2007;3:678-83.

14. Abood AA, Abdalla AN, Avakian SK. The application of multiagent technology on transient stability assessment of Iraqi super grid network. Am J Appl Sci 2008;5:1494-8. 
15. Dou C, Mao C, Bo Z, Zhang X. A multi-agent model based decentralized coordinated control for large power system transient stability improvement. In: 45th Int Univ Power Eng Conf (UPEC), 2010:1-5.

16. Dou C-X, Yang J, Li X, Gui T, Bi Y. Decentralized coordinated control for large power system based on transient stability assessment. Int J Electr Power Energy Syst 2013;46:153-62.

17. Karady G, Mohamed M. Improving transient stability using fast valving based on tracking rotor-angle and active power. In: IEEE Power Energy Soc Summer Meet, Vol. 3, 2002:1576-81.

18. Karady G, Daoud A, Mohamed M. On-line transient stability enhancement using multi-agent technique. In: IEEE Power Energy Soc Winter Meet, Vol. 2, 2002:893-99.

19. Mahmud MA, Pota HR, Hossain MJ. Full-order nonlinear observer-based excitation controller design for interconnected power systems via exact linearization approach. Int J Electr Power Energy Syst 2012;41:54-62.

20. Mahmud MA, Pota HR, Aldeen M, Hossain MJ. Partial feedback linearizing excitation controller for multimachine power systems to improve transient stability. IEEE Trans Power Syst 2014;29:561-71.

21. Rahman MS, Pota HR, Mahmud MA, Hossain MJ. A decentralised multi-agent approach to enhance the stability of smart microgrids with renewable energy. Int J Sustainable Energy 2014: 1-14. DOI:10.1080/14786451.2014.911741.

22. Rahman MS, Pota HR, Mahmud MA. Online transient stability improvement using distributed multi-agent scheme. In: IEEE 6th Int Conf Model Identif Control (ICMIC), 2014:1-6.

23. Rahman MS, Mahmud MA, Pota HR, Hossain MJ. A multi-agent approach for enhancing transient stability of smart grids. Int J Electr Power Energy Syst 2015;67:488-500.

24. Wang X, Zhang P, Wang Z, Dinavahi V, Chang G, Martinez J, et al. Interfacing issues in multiagent simulation for smart grid applications. IEEE Trans Power Delivery 2013;28:1918-27.

25. Baxevanos I, Labridis D. Implementing multiagent systems technology for power distribution network control and protection management. IEEE Trans Power Deliv 2007;22:433-43.

26. Phadke A, Thorp J. Synchronized phasor measurements and their applications. Ch. 9. New York, USA: Springer, 2008:197-221.

27. Roy NK, Pota HR, Hossain MJ. A cost-effective approach for fast voltage recovery of power distribution networks with renewable energy. Int J Sustainable Energy 2014;33:587-605.
28. Rahman MS, Mahmud MA, Pota HR, Hossain MJ. Distributed multi-agent scheme for reactive power management with renewable energy. Energy Convers Manage 2014;88:573-81.

29. Mithulananthan N, Salama MMA, Canizares CA, Reeve J. Distribution system voltage regulation and var compensation for different static load models. Int J Electr Eng Edu 2000;37:384-95.

30. Sadat H. Power system analysis. New York, USA: McGraw-Hill, 2002.

31. Sauer PS, Pai MA. Power system dynamics and stability. Stipes Publishing L.L.C: Champaign, IL-61820, 2006.

32. Mahmud M, Hossain MJ, Pota H, Ali M. Generalized Lyapunov function for stability analysis of interconnected power systems. In: 21st Australasian Univ Power Eng Conf (AUPEC), 2011:1-6.

33. Athay T, Podmore R, Virmani S. A practical method for the direct analysis of transient stability. IEEE Trans Power App Syst 1979;98:573-84.

34. Kakimoto N, Ohsawa Y, Hayashi M. Transient stability analysis of electric power systems via lure type Lyapunov function, part-I and II. IEE Trans Japan 1978;98:62-79.

35. Pai MA. Energy function analysis for power system stability. Ph. D. thesis, Kluwer Academic Publishers, University of Illinois at Champaign/Urbana, 1989.

36. Perez L, Flechsig A, Venkatasubramanian V. Modeling the protective system for power system dynamic analysis. IEEE Trans Power Syst 1994;9:1963-73.

37. Hahn A, Ashok A, Sridhar S, Govindarasu M. Cyber-physical security testbeds: architecture, application, and evaluation for smart grid. IEEE Trans Smart Grid 2013;4:847-55.

38. Rahman M, Pota H, Hossain MJ. Cyber vulnerabilities on agentbased smart grid protection system. In: IEEE Power Energy Soc Gen Meet, 2014:1-5.

39. Mahmud M, Hossain MJ, Pota H. Investigation of critical parameters for power systems stability with dynamic loads. In: IEEE Power Energy Soc Gen Meet, 2010:1-6.

Note: The preliminary version of this manuscript has been presented at IEEE International Conference on Modelling, Identification and Control 2014 (Manuscript Number: 89). 